\title{
Use of recombinant human parvovirus B19 antigens in serological assays
}

\author{
H A Cubie, E E Leslie, S Smith, H J O’Neill, H Hart, B J Cohen, J M Inglis
}

\begin{abstract}
Aims-To compare the sensitivity, specificity, and practicality of recombinant proteins in serological tests for the detection of human parvovirus B19 IgG and IgM.

Methods-Indirect enzyme linked immunosorbent assays using B19 structural proteins expressed in Escherichia coli were developed for the detection of B19 specific IgG and IgM (rELISA-G and rELISA-M). Cells infected with baculovirus expressing B19 structural proteins were also used in an indirect immunofluorescence assay for IgG and IgM antibodies (IFA-G and IFA-M). Antibody capture radioimmunoassays for IgG and IgM (GACRIA and MACRIA) were used as comparative assays.
\end{abstract}

Results-Twenty nine pools of intravenous immunoglobulin were clearly positive for B19 IgG by rELISA-G and contained low IgG titres by GACRIA. From 113 samples tested by all methods, sensitivities of $92 \%(77 / 84)$ and $97 \%$ (68/70) were obtained for ELISA and immunofluorescence, respectively, when compared with GACRIA. One hundred and sixteen samples from patients presenting with rash or arthralgia were compared by MACRIA, rELISA-M, and IFA-M. Sensitivities of both recombinant tests were more than $95 \%$. Despite pretreatment to remove IgG or rheumatoid factor, false positive results were a problem in the rELISA-M but were not seen with the IFA-M.

Conclusions-The limited supply of native antigen has severely restricted the wide application of serology for parvovirus B19. The use of recombinant antigens permitted the introduction of local screening tests which had many advantages, including quicker results and relief of the burden on the Reference Laboratory. The use of rELISA-M for sensitivity and IFA-M for specificity and confirmation proved a useful and practical combination for diagnosis of recent infection with B19, and rELISA-G allowed the immune response to be determined in selected populations.

(F Clin Pathol 1993;46:840-845)

Human parvovirus B19 is the causative agent of the common childhood infection, erythema infectiosum. B19 is also a major cause of aplastic crises ${ }^{1}$; there is also a $9 \%$ risk of fetal loss in infected pregnancy ${ }^{2}$; it can cause hydrops fetalis, especially with mid-trimester infection ${ }^{3}$; and recently, it has been shown to be the cause of persistent severe anaemia in immunocompromised individuals, including children with acute lymphatic leukaemia (ALL) and patients with HIV infection. ${ }^{45}$

Until recently the most useful serological tests for the detection of IgM antibodies to B19 were the anti- $\mu$ capture radioimmunoassay (MACRIA) ${ }^{6}$ or enzyme linked immunosorbent assay (ELISA). ${ }^{7-9}$ These tests, however, were established with antigen derived from the plasma or serum of viraemic patients and this has restricted their availability to a few centres. The lack of native antigen has also severely restricted the development of B19 IgG assays as screening tests for evidence of past infection. More recently, expression systems, both prokaryotic and eukaryotic, have been described ${ }^{10-14}$ and used for serological investigations. These test systems, based on immunofluorescence, $\mu$-capture, and indirect ELISA showed good correlation with MACRIA results. An evaluation of a commercially available ELISA using a B19 specific synthetic peptide, however, suggested lower sensitivity and specificity than MACRIA. ${ }^{15}$ Clearly more extensive comparisons and evaluations are required before alternatives to tests using native antigen are introduced more widely.

A baseline screen for newly diagnosed patients with leukaemia, chronic haemolytic anaemia, haemophilia, HIV infection, and for potential bone marrow transplant patients would be useful. Pregnant women exposed to B19 infection and laboratory staff working with specimens containing parvovirus should also be screened. ${ }^{2}$ Screening was carried out in a recently described outbreak of B19 infection in a paediatric ward and people at risk were excluded. ${ }^{16}$ Our aim was to produce tests for specific IgM and IgG with similar laboratory protocols such that they could be done at the same time, using minimum sample volume, and as economically as possible.

\section{Methods}

The following were tested for B19 IgG by ELISA: a panel of 13 sera consisting of six B19 IgG negative, three B19 IgG and IgM positive, and four B19 IgG positive samples; 29 batches of intravenous immunoglobulin, prepared by cold ethanol fractionation ${ }^{17}$ and

Correspondence to: Dr H A Cubie 
pH4 pepsin treatment, ${ }^{18}$ from pooled human plasma and stored lyophilised over several years by the Scottish National Blood Transfusion Service; and 113 sera submitted to the Regional Virus Laboratory, Edinburgh in 1991 for B19 IgG testing. During the first six months of 1992 , serum samples submitted for routine virological investigation of rash or arthralgia were tested simultaneously for B19 IgG and IgM by ELISA and 116 were available for comparative testing by MACRIA and IFA-M. Forty eight specimens shown to contain rubella, Epstein-Barr virus, cytomegalovirus, or hepatitis A IgM were tested for B19 rELISA-M and IFA-M.

For IgG testing (rELISA-G), microtitre modules (Nunc Immunostrips) were coated with $1 \mu \mathrm{g} / \mathrm{ml}$ of recombinant protein, derived from part of the VP1 gene expressed in $E$ coli (kindly provided by Mikrogen). The protein was diluted in freshly made $0 \cdot 1 \mathrm{M}$ carbonate buffer at pH9.6 and the strips held at $4^{\circ} \mathrm{C}$ overnight. They were washed three times with phosphate buffered saline containing $1 \%$ Tween 20 (PBST), blocked with PBST containing 3\% bovine serum albumin (BSA) for 1 hour at room temperature, washed three times with PBST, and dried. Strips could be sealed and kept at $4^{\circ} \mathrm{C}$ for at least one week without deterioration. A 1 in 100 dilution of serum in PBST $+1 \%$ BSA $(100 \mu \mathrm{l})$ was added to duplicate wells and incubated for 2 hours at $37^{\circ} \mathrm{C}$. The strips were washed five times with PBST and $100 \mu$ l anti-human conjugate labelled with alkaline phosphatase (Sigma, A3150; 1 in 800 in PBST $+1 \%$ BSA) was added and incubated for 1 hour at $37^{\circ} \mathrm{C}$. The strips were washed five times with PBST and $100 \mu \mathrm{l}$ para-nitro phenyl phosphate (Sigma, N9389) at $5 \mu \mathrm{g} / \mathrm{ml}$ in freshly made diethanolamine buffer (pH9.8) was added. The colour reaction was stopped after 30 minutes of incubation at room temperature with $100 \mu \mathrm{l} 3 \mathrm{M} \mathrm{NaOH}$. Absorbances were read at $405 \mathrm{~nm}$. A cutoff in test runs was taken as the mean absorbance of three different negative samples tested in duplicate plus 3 standard deviations from the mean $(+3 S D)$. A test:cutoff ratio ( $\mathrm{T}: \mathrm{CO})$ was calculated by dividing the mean of duplicate absorbances of the test specimen by the cutoff. A T:CO of 1 or greater was taken as positive; a T:CO between 0.9 and 1.0 was considered equivocal.

For IgM testing (rELISA-M), two structural proteins comprising the $\mathrm{N}$-terminal half of VP1 and the common C-terminal part of VP1/VP2 were used as a mixture with each diluted to $0.5 \mu \mathrm{g} / \mathrm{ml}$. Coating and blocking were carried out as for IgG tests. Sera were pretreated to remove IgG by adding $5 \mu \mathrm{l}$ of serum to $35 \mu \mathrm{l}$ of goat anti-human IgG ("GullSORB" reagent, Alpha Bioscan Ltd, Bognor Regis). After rota mixing and incubating for 15 minutes at room temperature the precipitate was removed by centrifugation at $3000 \mathrm{rpm}$ for 10 minutes in a bench centrifuge. The supernatant fluid was further diluted 1 in 10 in PBST (final dilution of 1 in 80 ). One hundred microlitres were added to three adjacent coated microtitre wells and incubated as for IgG estimations. IgM conjugate (Sigma, A3275; 1 in 800 in PBST + 1\% BSA) was added to two wells and IgG conjugate to the third well to check for IgG depletion. The test was completed under the same conditions as the IgG test and the T:CO were calculated as for rELISA-G except that the cutoff was set at Mean + 5SD.

For routine diagnostic samples, IgG and IgM estimations were carried out simultaneously. Separate IgG and IgM tests were carried out when appropriate.

For indirect immunofluorescence assay, insect cells infected with baculovirus recombinant expressing the VP1 protein of B19 were spotted on to glass slides. Slides were either fixed in methanol or by heat and acetone. The IFA was carried out essentially as described by Brown et al ${ }^{12}$ with the following minor modifications: blocking was carried out using veronal-buffered saline containing $1 \%$ guinea pig complement, and the same diluent was used to dilute sera; "GullSORB" was used as described above for the rELISA-M test; sheep anti-human IgG and IgM sera conjugated to fluorescein isothiocyanate (FITC) were provided by the Scottish Antibody Production Unit (Carluke) and slides were mounted in a $50 \%$ glycerol-phosphate buffer, pH8.4. IgG (IFA-G) and IgM (IFA-M) tests were routinely carried out at a serum dilution of 1 in 16 .

Antibody capture radioimmunoassays for B19 IgM (MACRIA) and IgG (GACRIA) were done as previously described. ${ }^{6}$ The results of RIA were expressed in arbitrary units (AU).

The presence of rheumatoid factor in a specimen was detected by latex agglutination (Wellcome Diagnostics). Interference from rheumatoid factor was reduced by pretreating the specimen with anti-human IgG and the absence of IgG in the treated sample was checked by ELISA. In some specimens it was removed by double absorption with heataggregated $\gamma$-globulin for 1 hour at $37^{\circ} \mathrm{C}$ and then overnight at $4^{\circ} \mathrm{C}$.

\section{Results}

ASSESSMENT OF RELISA-G

The 13 samples in the control panel from Germany were tested on many occasions without knowledge of their IgG status. The absorbances at $405 \mathrm{~nm}$ of these multiple runs are shown in the figure. The separation of negative and positive samples was clear cut, with most negative samples giving absorbances of 0.2 or less and most positive samples giving absorbances of 0.4 or greater.

Twenty nine samples of stored intravenous immunoglobulin plasma pools were tested in the rELISA-G. All samples gave a clearly positive T:CO $(>2.0$ for all samples on replicate testing). Dilution in PBST $+1 \%$ BSA, however, suggested that the titre of most samples was fairly low, with 21 giving negative ratios at a dilution of more than 1 in 800 . Low antibody titres were also 
Absorbances of control panel of sera (13 samples from Germany, labelled GP1-GP13) obtained by rELISA-G on different occasions.

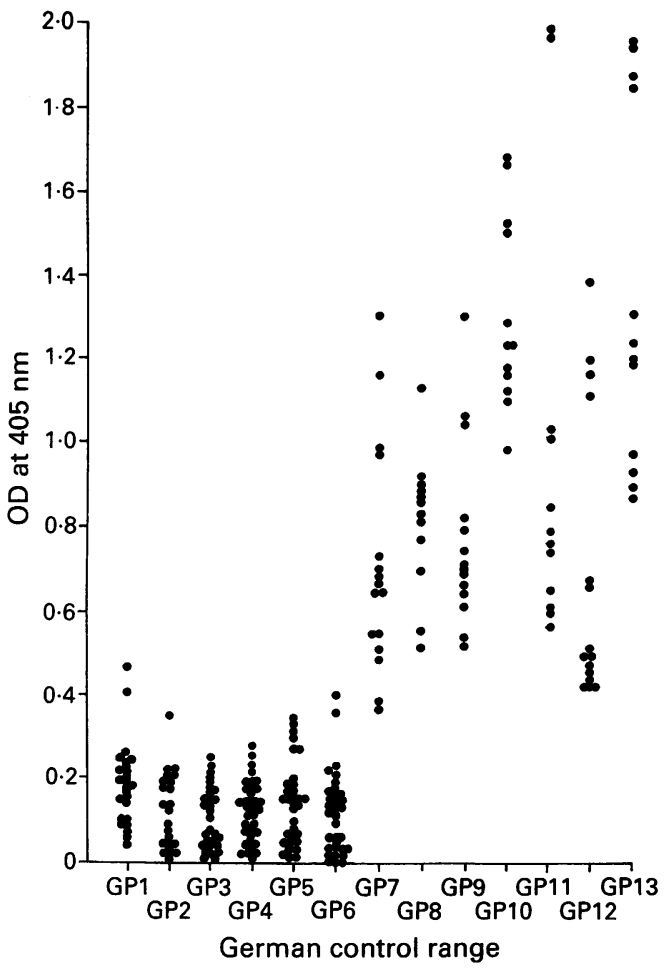

detected in all samples by GACRIA (4.5-16.0 AU).

\section{IgG BASELINE SCREENING}

One hundred and thirteen sera from patients presenting with rash and arthropathy were tested by rELISA-G and $79(70 \%)$ were positive. The results were compared with those obtained by GACRIA and IFA-G and are shown in table 1. Each test gave a few equivocal results. When these are included as positive and with GACRIA as the standard, the sensitivity and specificity of rELISA-G were $88 \%(77 / 86)$ and $83 \%(24 / 29)$, respectively. In the samples available for testing, a sensitivity of $97 \%$ and specificity of $100 \%$ was obtained for IFA-G.

Table 1 Comparison of results obtained by GACRIA on 113 specimens with results obtained by $r E L I S A-G$ and IFA-G

\begin{tabular}{|c|c|c|c|c|c|c|c|c|}
\hline \multirow[b]{2}{*}{$G A C R I A$} & & \multicolumn{3}{|c|}{$B 19 r E L I S A-G$} & \multicolumn{4}{|l|}{$I F A-G$} \\
\hline & & Positive & Equivalent & Negative & Positive & Equivalent & Negative & $N T$ \\
\hline $\begin{array}{l}\text { Positive } \\
\text { (>3 AU) }\end{array}$ & 75 & 72 & 0 & 3 & 63 & 0 & 0 & 12 \\
\hline $\begin{array}{l}\text { Equivocal } \\
\text { (1-3 AU) }\end{array}$ & 9 & 3 & 2 & 4 & 3 & 2 & 2 & 2 \\
\hline $\begin{array}{l}\text { Negative } \\
\text { Total }\end{array}$ & $\begin{array}{r}29 \\
113\end{array}$ & $\begin{array}{r}4 \\
79\end{array}$ & $\begin{array}{l}1 \\
3\end{array}$ & $\begin{array}{l}24 \\
31\end{array}$ & $\begin{array}{r}0 \\
66\end{array}$ & $\begin{array}{l}0 \\
2\end{array}$ & $\begin{array}{l}26 \\
28\end{array}$ & $\begin{array}{r}3 \\
17\end{array}$ \\
\hline
\end{tabular}

NT $=$ Not tested

Table 2 Comparison of results obtained on 116 specimens by $M A C R L A$, with results obtained by B19 rELISA-M and IFA-M

\begin{tabular}{|c|c|c|c|c|c|c|c|c|}
\hline \multirow[b]{2}{*}{$M A C R I A$} & & \multicolumn{3}{|c|}{$B 19 r E L I S A-M$} & \multicolumn{4}{|l|}{ IF $A-M$} \\
\hline & & Positive & Equivalent & Negative & Positive & Equivalent & Negative & $N T$ \\
\hline $\begin{array}{l}\text { Positive } \\
\text { (>3 AU) }\end{array}$ & 21 & 21 & 0 & 0 & 20 & 0 & 0 & 1 \\
\hline $\begin{array}{l}\text { Equivocal } \\
(1-3 \text { AU }\end{array}$ & 2 & 1 & 0 & 1 & 1 & 0 & 1 & 0 \\
\hline $\begin{array}{l}\text { Negative } \\
(<1 \text { AU })\end{array}$ & 93 & 24 & 0 & 69 & 0 & 3 & 82 & 8 \\
\hline Total & 116 & 46 & 0 & 70 & 21 & 3 & 83 & 9 \\
\hline
\end{tabular}

ASSESSMENT OF IgM rELISA-M AND IFA-M

One hundred and sixteen specimens from patients with a clinical history of rash or arthralgia were analysed by rELISA-M. These sera were also tested by MACRIA and IFA-M and the results are shown in table 2 . All 21 positive results detected by MACRIA were also positive by rELISA-M and the 20 which could be tested by IFA-M were also positive. No equivocal results were obtained by rELISA-M. When the MACRIA equivocal results were included, a sensitivity of more than $95 \%$ was obtained for both recombinant tests. The specificity of rELISA-M was $74 \%$ (69/93) and of IFA-M was $100 \%$ (85/85) for those samples which could be tested. Reproducible results were obtained both in the same test run and when tested on different occasions.

\section{EXAMINATION OF DISCREPANT SERA}

Further examination of 20 of the samples where IgM results disagreed was possible (table 3). Five (cases 1-5) were from samples which contained rubella specific IgM detected by ELISA and by SDG-HAI (sucrose density gradient separation followed by haemagglutination inhibition of the fractions). Three specimens contained rheumatoid factor which might have interfered in the reaction despite procedures to remove it. Four specimens came from young adult women who were pregnant, who had developed or been in contact with a rash, who had evidence of past infection with rubella and whose sera did not contain demonstrable rheumatoid factor. A further four were from non-pregnant adult women. Two specimens were from children with malignant disease (cases 17 and 18) and the remaining two specimens were from men (cases 19 and 20) one of whom had thalassaemia and the other who was immunocompromised and had a persisting B19 infection. The rELISA-M result from case 20 was negative while that from MACRIA was equivocal $(2 \cdot 2 \mathrm{AU})$.

CROSS-REACTIONS IN SERA WITH IgM SPECIFIC FOR OTHER VIRUSES

Testing of sera from patients with rashes which had already been examined for the presence of rubella specific IgM showed a number of anomalies. Of 25 rubella IgM negative sera, 22 were also B19 IgM negative, but the other three were B19 IgM positive by both rELISA-M and MACRIA, suggesting recent $\mathrm{B} 19$ infection. Of 32 rubella IgM positive sera, 21 were negative for B19 IgM by rELISA-M and 11 gave contradictory results (table 4). Further analysis showed two samples to be false positive by rubella indirect ELISA-M; one sample showed sequential infection with B19 and rubella and eight samples were positive by B19 rELISA-M but not by IFA-M nor by MACRIA. Four of these contained rheumatoid factor which was removed by the same method in both $B 19$ rELISA-M and IFA-M tests. Thus of 27 samples clearly positive for rubella IgM, eight gave false positive B19 rELISA-M results 
Table 3 Analysis of discrepant results by B19 rELISA-M, IFA-M, and MACRIA

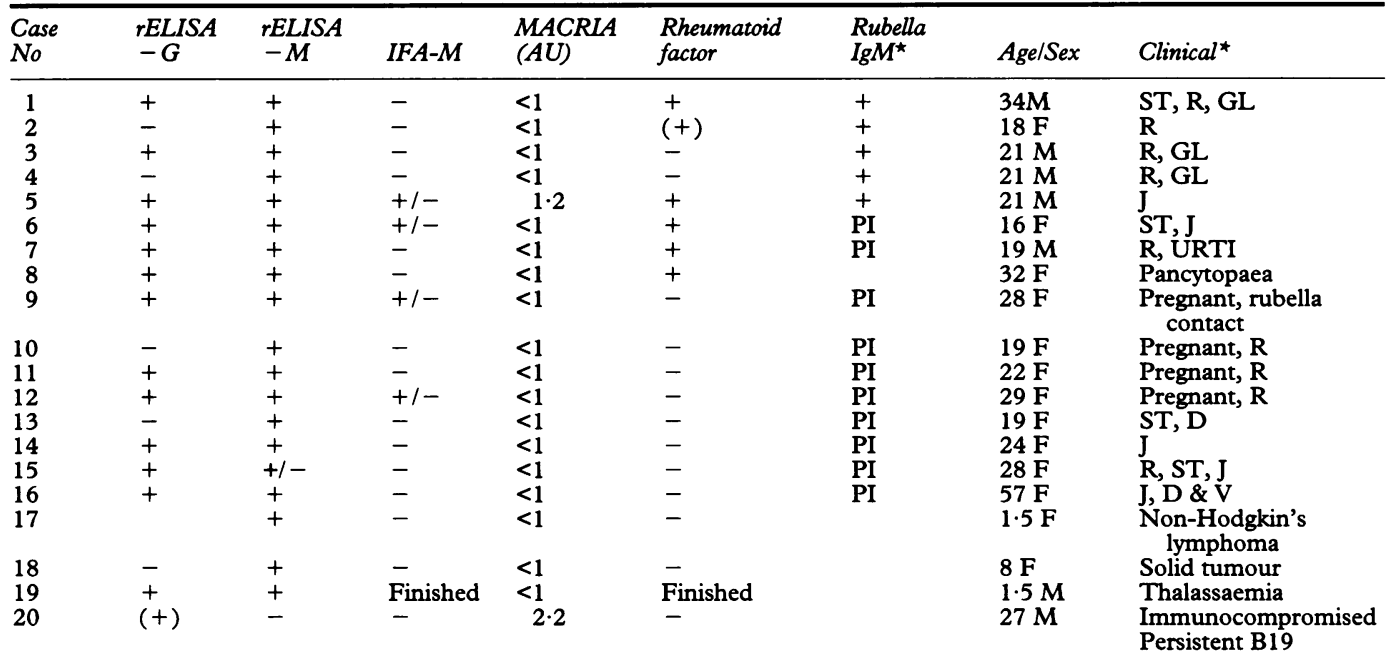

^Rubella IgM - tested by ELISA-M and SDG-HAI. PI = known past infection. GL = glands; $J=$ joints/arthralgia; $R=$ rash; $S T=$ sore throat; URTI = upper respiratory tract infection; D\&V = diarrhoea and vomiting.

which could not readily be explained by the presence of rheumatoid factor.

A further 16 sera containing IgM antibodies against Epstein-Barr virus, hepatitis A virus, or cytomegalovirus were then examined by B19 rELISA-M. Nine of these gave apparently false positive results (table 4), but rheumatoid factor was only present in five of them. One problem serum which had given IgM positive results in several IgM tests for rubella, cytomegalovirus, and Epstein-Barr virus was reproducibly negative in the B19 rELISA-M. None of these sera gave a positive result in B19 IFA-M.

\section{PRETREATMENT OF SERA}

The standard pretest procedure was to remove IgG using "GullSORB", and this was used for all IgM tests described above. Several combinations of pretreatment were investigated in an attempt to reduce the number of false positive rELISA-M results:

(a) Pretreatment with heat-aggregated $\gamma$ globulin The supernatant fluid, following the double absorption procedure, was diluted 1 in 80 in PBST + $1 \% \mathrm{BSA}$ and the ELISA carried out as before. All sera gave the same

Table 4 B19 rELISA-M positive results in 48 specimens containing specific IgM induced by other viruses

\begin{tabular}{|c|c|c|c|c|}
\hline \multirow[b]{2}{*}{ Virus } & \multirow[b]{2}{*}{$\begin{array}{l}\text { Antibody } \\
\text { response }\end{array}$} & \multicolumn{2}{|c|}{ Number of specimens } & \multirow[b]{2}{*}{ Comment } \\
\hline & & $\begin{array}{l}\text { Total } \\
\text { tested }\end{array}$ & $\begin{array}{l}\text { B19 rELISA-M } \\
\text { positive }\end{array}$ & \\
\hline \multirow[t]{2}{*}{ Rubella } & IgM + & 29 & 8 & \multirow{5}{*}{$\begin{array}{l}\text { B19 rELISA-M false + } \\
(4 \mathrm{RF}+; 4 \mathrm{RF}-) \\
\text { Rubella IgM false }+ \\
\text { Double sequential } \\
\text { infection` } \\
\text { B19 rELISA-M false + } \\
(2 \mathrm{RF}+; \text {; } \mathrm{RF}-) \\
\text { B19 rELISA-M false + } \\
(2 \mathrm{RF}+; 2 \mathrm{RF}-) \\
\text { B19 rELISA-M false +;RF + } \\
\text { False positive IgM } \\
\text { results in various } \\
\text { tests, but not B19 } \\
\text { rELISA-M; RF }+\end{array}$} \\
\hline & & $\begin{array}{l}2 \\
1\end{array}$ & $\begin{array}{l}2 \\
1\end{array}$ & \\
\hline EBV & $\operatorname{IgM}+$ & 6 & 4 & \\
\hline HAV & $\operatorname{IgM}+$ & 5 & 4 & \\
\hline $\begin{array}{l}\text { CMV } \\
\text { EBV, CMV } \\
\text { and } \\
\text { Rubella }\end{array}$ & $\underset{\text { IgM }+}{\operatorname{IgM}+}$ & $\begin{array}{l}4 \\
1\end{array}$ & $\begin{array}{l}1 \\
0\end{array}$ & \\
\hline
\end{tabular}

$\star$ See case 5 in table 3.

$\mathrm{EBV}=$ Epstein-Barr virus; $\mathrm{HAV}=$ hepatitis $\mathrm{A}$ virus; $\mathrm{CMV}=$ cytomegalovirus; $\mathrm{RF}=$ rheumatoid factor results as with "GullSORB" pretreatment whether or not B19 IgM positivity was specific or false.

(b) Combined absorption to remove IgG (standard "GullSORB" treatment) followed by removal of rheumatoid factor overnight at $4^{\circ} \mathrm{C}$ with heat-aggregated $\gamma$-globulin) Of 12 sera containing B19 specific IgM, one became negative and one equivocal after such treatment; of 25 problematic sera, 17 remained false positive, one gave a borderline positive result, and seven had become negative.

Neither (a) nor (b) significantly improved the specificity and the standard pretreatment procedure has therefore been retained for future testing.

\section{Discussion}

The B19 ELISAs were easy to perform and gave reproducible results both within assays and from day to day. The antigens supplied by Mikrogen were stable at $4^{\circ} \mathrm{C}$ and have now been in use for up to 18 months without deterioration. Minimum sample volume was required ( $3 \mu \mathrm{l}$ of serum for rELISA-G and $5 \mu \mathrm{l}$ for rELISA-M). Reagent costs have been estimated to be less than $70 p$ for duplicate testing and the rELISA-G could therefore offer a simple, inexpensive screening test which in our sample gave good agreement with GACRIA and IFA-G. The recent development of commercially available assays using recombinant proteins should allow much more extensive comparisons in the future. In the current study $70 \%$ of sera studied gave positive results by rELISA-G, a finding comparable with the $60 \%$ of blood donors shown to be positive by GACRIA ${ }^{6}$ and the $76 \%$ of blood donors positive by IFA-G. ${ }^{12}$

Other workers have estimated the sensitivity of their B19 IgG testing by looking for rises in antibody titre. Thus Erdman et al ${ }^{11}$ detected $87 \%$ of acute B19 infections using a capture ELISA with B19 antigen expressed on Chinese hamster ovary cells on paired sera, and Patou and Ayliffe ${ }^{15}$ detected $67 \%$ of 
such infections using a synthetic peptide in an indirect ELISA. There seems little value in using an ELISA-G test diagnostically on paired specimens when a suitable IgM test is available, but there is a place for screening for past infection with B19 in selected groups of people such as the immunocompromised before treatment, pregnant women in contact with suspected B19 infection, and laboratory workers handling infectious specimens containing B19.

One of the problems associated with the use of native antigens is that they are infectious. ${ }^{1920}$ Cohen and Brown recommend $\gamma$ irradiation to inactivate B19 antigen, containment of potential aerosols within a class I cabinet, and the monitoring of laboratory staff for immunity. ${ }^{20}$ The problem of laboratory acquired infection highlights the need for antibody assays based on recombinant or synthetic peptide antigens.

It would be useful to have a simple, inexpensive B19 IgG test to allow more extensive population and follow up studies to be carried out than has been possible to date. On a quantitative basis it would also be useful for estimating the content of B19 specific IgG in batches of intravenous immunoglobulin suitable for treatment of immunocompromised patients with persistent B19 infection. The samples tested here were more strongly positive by rELISA-G (all had a T:CO ratio of more than 2) than by GACRIA (all values under $16 \mathrm{AU}$ ). Quantification, however, requires further study and the use of international standards for controls.

The rELISA-M was also easy to perform and could be carried out at the same time as rELISA-G to give results indicative of both current or past infection. Simultaneous assays for both IgG and IgM antibodies have proved useful in other virus infections, such as Epstein-Barr virus where knowledge of both results can influence further testing and advice. Our r-ELISA-M protocol, however, starts with the removal of IgG from the specimen using commercially available antibody to human IgG and this adds significantly to its cost, more than doubling the cost of our inhouse rELISA-G.

The rELISA-M showed a sensitivity of $95 \%$ and a specificity of $74 \%$ when compared with MACRIA. False positive results, particularly in sera containing IgM against antigens from other viruses, presented a problem. Modification of the pretreatment of sera to include removal of rheumatoid factor as well as IgG reduced the number of false positive results but would have caused one of 12 true positive results to have been missed. It is clear, however, that the false positive results are not simply due to rheumatoid factor because fewer than half the problem sera in the present study seemed to contain rheumatoid factor, nor are they due to interference from residual IgG because removal of IgG is monitored by incubating with IgG conjugate after absorption. The explanation for these false positive results remains to be found but this is a recognised problem in indirect assays and this has been noted by other workers, particularly when rheumatoid factor negative, rubella IgM containing sera were examined..$^{91}$ The use of antibody capture assays can reduce the problem of false positive results considerably. Recent experience in our own laboratory with commercially available indirect and $\mu$-capture ELISA tests for rubella, however, have shown a loss of sensitivity at the expense of specificity (unpublished observations).

In another study Brown and colleagues found cross-reactivity in 24 sera containing rubella specific IgM which was apparently not evident when indirect immunofluorescence was used. ${ }^{12}$ In the study reported here rELISA-M false positive results were negative when tested by IFA-M on cells expressing VPI from a baculovirus vector (tables 2 and 3 ), showing the greater specificity of the IFAM. Positive results on duplicate testing by rELISA-M, matched with clearly positive results by IFA-M, have also always been MACRIA positive. There were three sera which gave borderline IFA-M positive results in the presence of MACRIA results of less than $1 \mathrm{AU}$ (table 3), but whether these represent false positive results by rELISA-M and IFA-M or false negative results by MARCIA is uncertain.

The continued provision of a regular and adequate supply of quality assured slides for IFA is currently being undertaken. Together with the purchase of tested recombinant B19 antigen for ELISA, this would enable B19 serology to be undertaken locally. Such testing would relieve the burden on the Reference Laboratory, which could still provide reference assays with native $B 19$ antigens when required. Local testing would also ensure a quicker and cheaper service to the clinicians and would enable outbreaks to be recognised earlier because of the closer contact between the reporting laboratory and the clinicians. With the advent of purchaser/ provider services such local arrangements will be valuable in the future.

In conclusion, we suggest that the B19 ELISA described here, using recombinant proteins to detect both IgG and IgM and giving results within one day, would be suitable for use at a local level and that the provision of IFA slides with insect cells expressing B19 proteins would provide a confirmatory assay suitable for smaller numbers of samples.

The initial provision of slides bearing B19 expressing insect cells by Dr Marcel Salimans, Leiden, sparked this investigation, with further slides being supplied by Dr Nigel Connor who was then employed by Northumbria Biologicals Ltd. The $E$ coli recombinant proteins came from $\mathrm{Dr} M$ Motz, Mikrogen, Munich. These gifts are acknowledged with thanks.

Control sera were kindly provided by Dr T Schwarz, Max van Pettenkofer Institute, Munich (B19 IgG Control panel). Thanks are due to Mrs Pamela Munro for typing the manuscript.

This work was supported by a grant for consumables from Lothian Health Board Research Allocations for 1991-92. 
1 Pattison JR, Jones SE, Hodgson J, et al. Parvovirus infections and hypoplastic crisis in sickle cell anaemia. Lancet t981;i:664-5.

2 Public Health Laboratory Service Working Party on Fifth Disease. Prospective study of human parvovirus (B19)

3 Brown T, Anand A, Ritchie LD, Clewley JP, Reid TMS Intrauterine parvovirus infection associated with hydrops foetalis. Lancet 1984;ii:1033-4.

4 Kurtzman GJ, Ozawaa K, Cohen B, Hansan G, Oseas R, Young NS. Chronic bone marrow failure due to persistent B19 parvovirus infection. N Engl f Med 1988; 317:287-94.

5 Frickhofen N, Abkowitz JL, Safford M, et al. Persistent B19 parvovirus infection in patients infected with human immunodeficiency virus-1: A treatable cause of humaemia in AIDS. Ann Intern Med 1990;113:926-33.

6 Cohen BJ, Mortimer PP, Pereira MS. Diagnostic assays with monoclonal antibodies for the human serum parvowith monocional antibodies for the human serum parvo-

7 Anderson LJ, Tsou C, Parker CA, et al. Detection of antiAnderson LJ, Tsou C, Parker CA, et al. Detection of antibodies and antigens of human parvovirus B19 by enzyme-linked imm

8 Schwarz T, Roggendorf M, Deinhardt F. Human parvovirus B19: ELISA and immunoblot assays. $f$ Virol Methods 1988;20:155-68.

9 O'Neill HJ, Coyle PV. Two anti-parvovirus B19 IgM capture assays incorporating a mouse monoclonal antibody V Virol 1992;123:125-34.

10 Kajigaya S, Shimada T, Fujita S, Young NS. A genetically engineered cell line that produces empty capsids of B19 (human) parvovirus. Proc Natl Acad Sci USA 1989;86: 7601-5.

11 Erdman DD, Usher MJ, Tsou C, et al. Human parvovirus
B19 specific IgG, IgA and IgM antibodies and DNA in serum specimens from persons with erythema infectiosum. 7 Med Virol 1991;35:110-15.

12 Brown CS, Van Bussel MJAWM, Wassenaar ALM, Van Elsacker-Niele AMW, Weiland HT, Salimans MMM An immunofluorescence assay for the detection of An immunofluorescence assay for the detection of parvovirus B19 IgG and IgM antibodies based on recom $53-62$.

13 Morinet F, Courouce AM, Galibert F, Perol Y. The use of labelled fusion protein for detection of B19 parvovirus IgM antibodies by an immunocapture test. $\mathcal{f}$ Viro Methods 1991;32:21-30.

14 Soderland $M$, Brown $K E$, Meurman $O$, Hedman $K$ Prokaryotic expression of a VPI polypeptide antigen for diagnosis by a human parvovirus B19 antibody enzyme immunoassay. 7 Clin Microbiol 1992;30:305-11.

15 Patou G, Ayliffe U. Evaluation of commercial enzyme linked immunosorbent assay for detection of B19 parlinked immunosorbent assay for detection of B19

16 Pillau D, Paton G, Hurt S, Kibbler CC, Griffiths PD. Parvovirus B19 outbreak in a children's ward. Lancet 1992;339:107-9.

17 Welch AG, Macintosh RV, Foster PR. Human Immunoglobulin for clinical use. Lancet $1983 ; \mathrm{i}: 358$

18 Reid KG, Cuthbertson B, Jones ADL, MacIntosh RV. The potential contribution of mild pepsin treatment at pH4 to the viral safety of human immunoglobulin products. Vox Sang 1988;55:75-80.

19 Shiraishi $H$, Sasaki $T$, Nakamura $M$, Yaegashi $N$, Sugamura $K$. Laboratory infection with human parvovirus B19. F Infect 1991;22:308-10.

20 Cohen BJ, Brown KE. Laboratory infection with human parvovirus B19. F Infect 1992;24:113-4.

21 Kurtz JB, Anderson MJ. Cross-reactions in rubella and parvovirus specific IgM test. Lancet 1985;ii:1356. 